\title{
Linear Transformation Recognition Using Radon Transform
}

\author{
Fawaz Hiouj \\ Department of Mathematics, East Carolina University, Greenville, NC 27858-4353, USA \\ Email: hioujf@ecu.edu.Phone: 252-876-3241
}

\begin{abstract}
Given two regular functions (images) $f$ and $g$ on $R^{2}$ where $g$ is formed from $f$ by a general linear transformation, $g(x)=f(A x+b)$. We present a procedure to determine the transformation 'parameters' $A$ and $b$ using Radon projections of $f$ and only two projections of $\mathrm{g}$. We use these projections together with simple facts on matrix vector multiplication to recover the matrix $A$. The assumptions we have here are: $f$ is nonnegative and $A$ is nonsingular. Commonly used transformations in image processing such as rotation, scaling and others are special cases of our approach.
\end{abstract}




\section{Introduction}

The question of image matching is one of the basic image processing concepts due to its many applications in detecting changes in a scene, estimating object motion, studying an inspected image, integrating information from different types of image, etc.

In this paper we present a method for recovering 2D affine transformations or a general linear transformation that relates an input distorted image to a known reference image. To some degree, this work is a generalization of the work we presented in [1], where we considered the class of images under possible distortion of rotation, scaling, reflection and translation.

These common geometrical transformations of images have been the target for many authors; for instance [1-4]. On the other hand, there is a growing body of studies on the more general cases of affine transformations, general linear transformations, or bilinear transformations. One finds different algorithms that are based on different implementations such as methods of moments, correlations, integral transformations, and others. For examples, [5] deals with the 2D affine transformation using a regular grid of points extracted from the distorted image and then compares it with a reference grid. The method of moments is often used, such as Zernike Moments [6], and Krawtchouk moments [7]; the trace transform method is used in [8]. These are just samples of the important work on this problem. This mathematical problem gains some of its importance due the connection with the engineering watermarking practice. However, according to [9], in signal processing there are no efficient algorithms known for the class of affine transformations.

Our method is based on using the relation between Radon transform of the images $f$ and $g$ together with the fact that a matrix $A$ maps the standard unit vector $\mathbf{i}=\left[\begin{array}{l}1 \\ 0\end{array}\right]$ to the first column of $A$ and the standard unit vector and $\mathbf{i}=\left[\begin{array}{l}0 \\ 1\end{array}\right]$ to the second column of $A$. We then use these tools to build possible solutions (matrices). Each candidate (matrix) is tested by a direct matching in the transform domain or in the function domain.

In the remaining part of this introduction we introduce notation and basic formulas. Suppose that $f$ is a 2-D function and $\mathbf{x}=\left(\begin{array}{l}x \\ y\end{array}\right) \in \mathbf{R}^{2}$. We will assume that the centroid of $f$ coincides with the origin. Given the unit vector

$\xi=(\cos \theta, \sin \theta)^{\mathrm{T}}$

The Radon Transform of $f$ along the line $L=\{(x, y): x \cos \theta+y \sin \theta=p\}$ is given by:

$f^{\vee}(p, \theta)=\int_{-\infty}^{\infty} f(p \cos \theta-t \sin \theta, p \sin \theta+t \cos \theta) d t$

We will be using the notation $f^{\vee}(p, \theta)$ and $f^{\vee}(p, \xi)$ interchangeably. Let $A$ be a $2 \times 2$ nonsingular matrix. Define the function

$g(\mathbf{x})=f(A \mathbf{x})$

Then, $\quad g^{\vee}(p, \xi)=\frac{|\operatorname{det}(B)|}{|\zeta|} f^{\vee}\left(\frac{p}{|\zeta|}, \frac{\zeta}{|\zeta|}\right)$ 
as shown in [10], where $B=A^{-1}$ and $\zeta=B^{T} \xi$, which is not in general a unit vector. Figure 1 shows an image illustration of a function $f$ and the function $g$ obtained by a linear transformation.

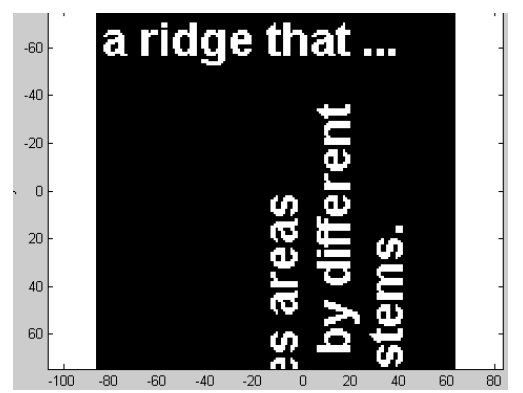

a

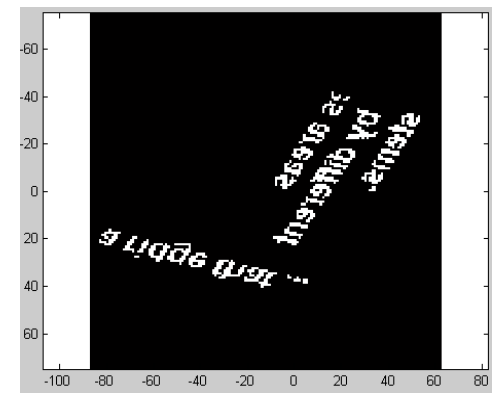

b

Figure1.a, b: A linear transformation of a text image.

\section{THE FORM $g(\mathbf{x})=f(A \mathbf{x})$}

Our goal is to recover the matrix $C:=B^{T}$ from (4). We begin with rewriting (4) in the scalar form: Let $\theta$ and $\varphi$ be the two angles that define the vectors $\xi$ and $\zeta$. i.e $\xi=(\cos \theta, \sin \theta)^{\mathrm{T}}$, and $\zeta=C \xi=|\zeta| .(\cos \varphi, \sin \varphi)^{\mathrm{T}}$.

(5)

We will be using these notations and relations throughout the discussion. Now, we write (4) in the form

$g^{\vee}(p, \theta)=\frac{|\operatorname{det}(C)|}{|\zeta|} f^{\vee}\left(\frac{p}{|\zeta|}, \varphi\right)$

From (6) it is possible then to show that

$$
|\operatorname{det} C|=\frac{\int_{p=-\infty}^{\infty} g^{\vee}(p, \varphi) d p}{\int_{p=-\infty}^{\infty} f^{\vee}(p, \theta) d p}
$$

with any choice of $\theta$ and $\varphi$.

The difficulty in using (4) or (6) with $C$ being unknown is that we need to know which angle $\theta$ would correspond to which angle $\varphi$. In trying to address this issue we develop some analytical formulas and arguments that will eventually be implemented numerically. Also, our approach offers the option of using only two projections of the inspected image $g$, namely angles $\theta=0$, and $\theta=\frac{\pi}{2}$, as will be explained shortly. Notice that if we square both sides of (6) we can obtain the useful relation: 


$$
|\zeta|=(|\operatorname{det} C|)^{2} \cdot \frac{\int_{p=-\infty}^{\infty}\left[f^{\vee}\right]^{2}(p, \varphi) d p}{\int_{p=-\infty}^{\infty}\left[g^{\vee}\right]^{2}(p, \theta) d p}
$$

In addition, for a choice of $\theta$ and $\varphi$ that match through (6) it would obviously be true that

$$
\left\|g^{\vee}(p, \theta)-\frac{|\operatorname{det}(C)|}{|\zeta|} f^{\vee}\left(\frac{p}{|\zeta|}, \varphi\right)\right\|_{2}=0
$$

where $\|\cdot\|_{2}$ is the 2-norm of a function. In some special cases it is possible to use merely (7) and (8) to recover the matrix of transformation. For a quick example: If the matrix $A$ represents a non uniform scaling. i.e. $A=\left[\begin{array}{ll}\mathrm{a} & 0 \\ 0 & \mathrm{~b}\end{array}\right]$ where a and $\mathrm{b}$ are non zero nonnegative real numbers, then $C$ would also be a diagonal matrix of the form $C=\left[\begin{array}{ll}\alpha & 0 \\ 0 & \beta\end{array}\right]$ for some $\alpha$ and $\beta>0$. We apply (7) and (8) with $\xi=(\cos 0, \sin 0)^{\mathrm{T}}$, from which we have $\theta=0, \quad \varphi=0, \quad \zeta=\mathrm{C} \xi$, and

$$
|\alpha|=|\zeta|=(|\operatorname{det} C|)^{2} \cdot \frac{\int_{p=-\infty}^{\infty}\left[f^{\vee}\right]^{2}(p, 0) d p}{\int_{p=-\infty}^{\infty}\left[g^{\vee}\right]^{2}(p, 0) d p}
$$

and $\beta=\frac{|\operatorname{det}(C)|}{\alpha}$. Figure (2) is an illustration of such a transformation using $A=\left[\begin{array}{lr}900 & 0 \\ 0 & 1.5000\end{array}\right]$ that can be recovered precisely using (10).

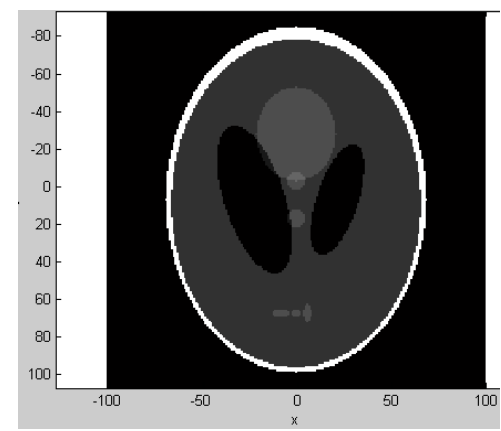

Figure $2 a$

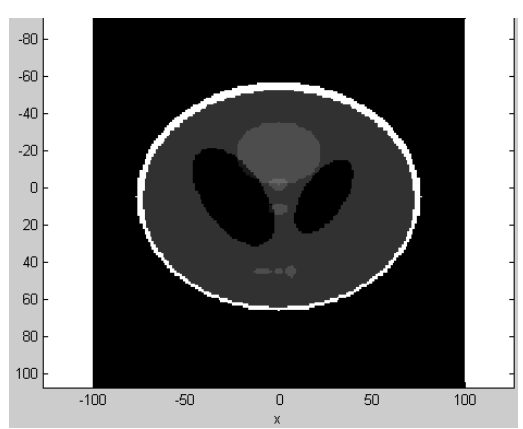

Figure $2 b$

Figure 2 a,b: Nonuniform scaling that can be recovered using (10)

Back to the concern of matching an angle $\theta$ to an angle $\varphi$. Each angle $\theta$ is associated (matched) with at least one unknown pair of an angle $\varphi$ and a real number $|\zeta|$ so that (6), (7), 
(8), and (9) are all satisfied. The values we need for the angle $\theta$ are 0 and $\frac{\pi}{2}$ that define the standard unit vectors $\mathbf{i}=\left[\begin{array}{l}1 \\ 0\end{array}\right]$ and $\mathbf{i}=\left[\begin{array}{l}0 \\ 1\end{array}\right]$. It is known that a matrix $C$ maps the vector $\mathbf{i}$ to the first column of $C$ and $\mathbf{i}$ to the second column of $C$. If $\varphi_{1}^{*}$ and $\varphi_{2}^{*}$ are the two angles that match with the angles $\theta=0$ and $\theta=\frac{\pi}{2}$ through (6), then we may express the two columns of $C$ in the forms:

$$
\begin{aligned}
& \zeta_{1}=C\left[\begin{array}{l}
1 \\
0
\end{array}\right]=\left|\zeta_{1}\right| \cdot\left(\cos \varphi_{1}^{*}, \sin \varphi_{1}^{*}\right)^{\mathrm{T}} . \\
& \zeta_{2}=C\left[\begin{array}{l}
0 \\
1
\end{array}\right]=\left|\zeta_{2}\right| \cdot\left(\cos \varphi_{2}^{*}, \sin \varphi_{2}^{*}\right)^{\mathrm{T}} .
\end{aligned}
$$

Motivated by (9); we define the functions:

$$
\begin{aligned}
& F_{0}(\varphi)=\left\|g^{\vee}(p, 0)-\frac{|\operatorname{det}(C)|}{|\zeta|} f^{\vee}\left(\frac{p}{|\zeta|}, \varphi\right)\right\|_{2} \\
& F_{\frac{\pi}{2}}(\varphi)=\left\|g^{\vee}\left(p, \frac{\pi}{2}\right)-\frac{|\operatorname{det}(C)|}{|\zeta|} f^{\vee}\left(\frac{p}{|\zeta|}, \varphi\right)\right\|_{2} \\
& -\pi \leq \varphi \leq \pi \text {; and }|\zeta| \text { as in (8) with } \theta=0, \frac{\pi}{2} \text { respectively. }
\end{aligned}
$$

Clearly, $F_{0}\left(\varphi_{1}^{*}\right)=0=F_{\frac{\pi}{2}}\left(\varphi_{2}^{*}\right)$. Notice that there is possibility for (a false match) i.e. It is possible to find more than one zero of $F_{0}, F_{\frac{\pi}{2}}$ or both. But our minimization process will be counting for these possibilities as we explain now. Define the matrix function $M$ of two columns $\zeta_{1}$ and $\zeta_{2}$ :

$M=M\left(\varphi_{1}, \varphi_{2}\right)=\left[\zeta_{1} \mid \zeta_{2}\right]$

$\left(\varphi_{1}, \varphi_{2}\right) \in[-\pi, \pi] \times[-\pi, \pi]$, where

$\zeta_{1}=\left|\zeta_{1}\right| \cdot\left(\cos \varphi_{1}, \sin \varphi_{1}\right)^{\mathrm{T}}, \quad\left|\zeta_{1}\right|$ as in (8) with $\varphi=\varphi_{1}, \theta=0$.

$\zeta_{2}=\left|\zeta_{2}\right| \cdot\left(\cos \varphi_{2}, \sin \varphi_{2}\right)^{\mathrm{T}},\left|\zeta_{2}\right|$ as in (8) with $\varphi=\varphi_{2}, \theta=\frac{\pi}{2}$.

Using (13) we now can define a reasonable class of matrices for a possible solution of the problem.

In view of the above discussion we define our objective function on $[-\pi, \pi] \times[-\pi, \pi]$ :

$$
\Psi_{1}\left(\varphi_{1}, \varphi_{2}\right)=\int_{\theta=0}^{\pi} \int_{p=-\infty}^{\infty}\left|g^{\vee}(p, \theta)-\frac{|\operatorname{det} M|}{|\zeta|} f^{\vee}\left(\frac{p}{|\zeta|}, \varphi\right)\right| d p d \theta
$$


where $\theta$ and $\varphi$ are the two angles that define the vectors

$$
\xi=(\cos \theta, \sin \theta)^{\mathrm{T}}, \zeta=M \xi=|\zeta|(\cos \varphi, \sin \varphi)^{\mathrm{T}} \text { and } M \text { from (13). Clearly, }
$$

$$
\Psi_{1}\left(\varphi_{1}, \varphi_{2}\right) \geq 0 \text { and } \Psi_{1}\left(\varphi_{1}{ }^{*}, \varphi_{2}{ }^{*}\right)=0 \text {. }
$$

We also can test possible matrices in the function domain by working with the objective function:

$$
\Psi_{2}\left(\varphi_{1}, \varphi_{2}\right)=\|g(\mathbf{x})-f(M \mathbf{x})\|_{2}
$$

where $g$ from (3) and $M$ from (13).

We will numerically find $\left(\varphi_{1}, \varphi_{2}\right) \in[-\pi, \pi] \times[-\pi, \pi]$ that minimizes $\Psi_{1}$ or $\Psi_{2}$. More on the numerical implementation is presented in the discussion section.

The Form $g(\mathbf{x})=f(A \mathbf{x}+\mathbf{b})$

Consider the function

$g(\mathbf{x})=f(A \mathbf{x}+\mathbf{b})$

$\mathbf{b} \in \mathbf{R}^{2}$. Let $\left(\frac{\bar{x}}{\bar{y}}\right)$ be the centroid of image $g$ :

$$
\bar{x}=\frac{\int_{-\infty}^{\infty} p g^{\vee}(p, 0) d p}{\int_{-\infty}^{\infty} g \vee(p, 0) d p} ; \bar{y}=\frac{\int_{-\infty}^{\infty} p g^{\vee}\left(p, \frac{\pi}{2}\right) d p}{\int_{-\infty}^{\infty} g \vee\left(p, \frac{\pi}{2}\right) d p}
$$

(17)

as derived in [1]. Given our assumption that the centroid of $f$ is the origin, a direct calculation of this center of mass shows that

$$
\mathbf{b}=-A\left(\begin{array}{l}
\bar{x} \\
\bar{y}
\end{array}\right)
$$

Define a new function, an image, whose centroid is the origin:

$g_{1}(x, y):=g(x+\bar{x}, y+\bar{y})=f\left\lfloor A\left(\begin{array}{l}x+\bar{x} \\ y+\bar{y}\end{array}\right)+\mathbf{b} \quad\right.$ - Using (18) we write,

$g_{1}(\mathbf{x})=f(A \mathbf{x})$

(19)

Thus, we need to work with (19) to obtain the matrix as already presented in the previous section.

\section{Discussion \& Remarks}

We want to select the matrix $C=B^{T}$ from a reasonable class of matrices $M$ that is defined in (13). We then test $M$ in the transform domain as in (14) or in the function domain as in (15). We conducted several tests on different types of images. For example, in figure $3 a, b$ we use the matrix $A=\left[\begin{array}{ll}1 & 2 \\ 3 & 4\end{array}\right]$ to make the transformation. Here, $C=\left[\begin{array}{cc}-2 & 1.5 \\ 1 & -.5\end{array}\right]$ with $\varphi_{1}^{*}=2.6779$ and $\varphi_{2}^{*}=-0.3218$. In figure $3 c, d$, we show the graphs of $F_{0}$, and $F_{\frac{\pi}{2}}$ on $[-\pi, \pi]$. The two minimizers of $F_{0}$, and $F_{\frac{\pi}{2}}$ are $\varphi_{1}^{*}$ and $\varphi_{2}^{*}$. 


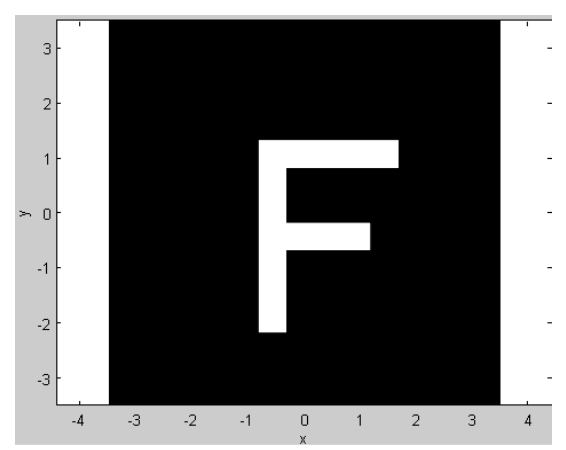

Figure $3 a$

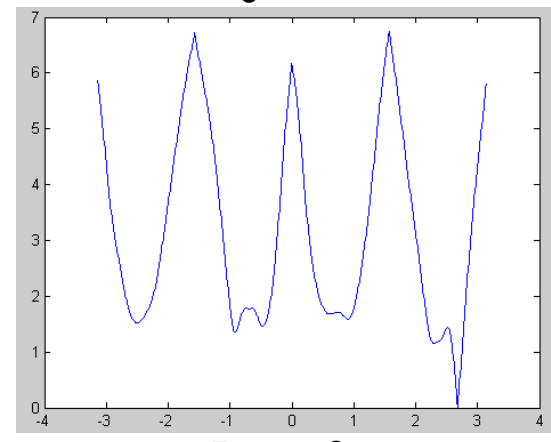

Figure $3 c$

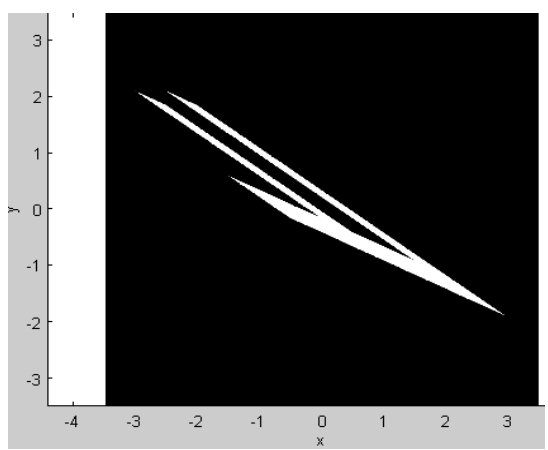

Figure $3 b$

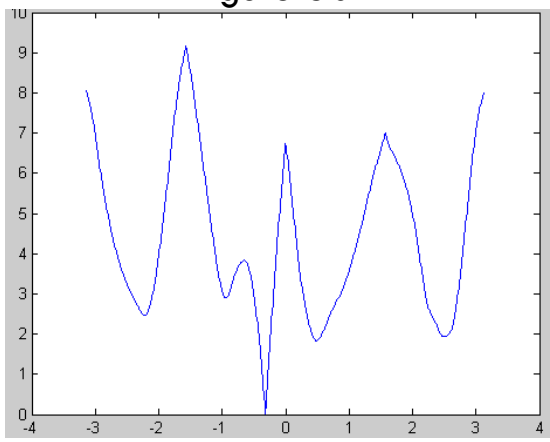

Figure 3d

Figure 3a, b: a block image and a linear transformation with $A=\left[\begin{array}{ll}1 & 2 \\ 3 & 4\end{array}\right]$

Figure 3c, d: $F_{0}\left(\varphi_{1}\right)$ from (11) and $F_{\frac{\pi}{2}}\left(\varphi_{2}\right)$ from (12) for the above transformation.

In devising this minimization process we make the following remarks:

First: We notice that if we work with (14), the values of the objective function $\Psi_{1}$ would be well separated compared to the values of $\Psi_{2}$. Second: in minimizing $\Psi_{1}$ or $\Psi_{2}$ we use the constraints that for every $\left(\varphi_{1}, \varphi_{2}\right) \in[-\pi, \pi] \times[-\pi, \pi], M$ is nonsingular, and both $F_{0}\left(\varphi_{1}\right)$ and $F_{\frac{\pi}{2}}\left(\varphi_{2}\right)$ in (1 1 ) and (12) must be substantially small as suggested by figures $3 c$, $d$.

Third: for testing our equations in a numerical setting we used the class of block images developed in [1], as the $F$ block in figure 3 . We also extend this approach to any $M \times N$ image. Indeed, we employ the known idea of representing an image as the sum of shifted boxes that we describe briefly: Let

$$
b(x, y):= \begin{cases}1 & \text { if } 0<\mathrm{x}<1 \text { and } 0<\mathrm{y}<1 \\ 0 & \text { otherwise. }\end{cases}
$$

be the unit square function whose Radon transform $b^{\vee}(p, \varphi)$ is known exactly [10]. Let $f$ be an $\mathrm{M} \times \mathrm{N}$ image, we work with the pixel and spatial coordinate systems simultaneously to write:

$$
f(x, y)=\sum_{i=1}^{\mathrm{M}} \sum_{j=1}^{N} b(x-j+1, y-M+i) \cdot f(i, j)
$$

Applying the translation rule of Radon transform we obtain:

$$
f^{\vee}(p, \varphi)=\sum_{\mathrm{i}=1}^{\mathrm{M}} \sum_{j=1}^{N} b^{\vee}\{p-[(\mathrm{j}-1) \cos \varphi+(M-i) \sin \varphi], \varphi\} . f(i, j)
$$


which is a way of computing the Radon transform of an image at any angle of projection. Forth: our method can be adjusted to handle some special cases of transformations. For example, the case of nonuniform scaling that we derived in (10). Another typical example is recovering a matrix of rotation: if $A$ is merely a rotation matrix then matrix $C$ is orthogonal which means that $\varphi_{1}^{*}=\varphi_{2}^{*} \pm \frac{\pi}{2}$ in which case we can think of $\Psi_{1}$ and $\Psi_{2}$ as functions in one variable on $[-\pi, \pi]$.

\section{Conclusion}

In this paper, we presented a method for identifying a linear transformation applied on an image. We compared the two functions $f$ and $g$ where $g$ is obtained by subjecting $f$ to a linear transformation: $g(\mathbf{x})=f(A \mathbf{x}+\mathbf{b})$. We used the relation between the Radon transforms of $f$ and $g$ to approximate the two columns of the matrix $C=B^{T}$ where $B=A^{-1}$. We also used the Radon transform in computing the center of mass of the inspected image that leads to compute the translation parameters.

\section{References}

[1] Hjouj F, Kammler DW, Identification of reflected, scaled, translated, and rotated objects from their radon projections, IEEE Trans Image Process. 2008 Mar; 17(3):301-10.

[2] Jafari-Khouzani K, Soltanian-Zadeh H.Rotation-invariant multiresolution texture analysis using radon and wavelet transforms, IEEE Trans Image Process. 2005 Jun; 14(6):783-95.

[3] Kokare $M$, Biswas PK, Chatterii BN. Texture image retrieval using new rotated complex wavelet filters, IEEE Trans Syst Man Cybern B Cybern. 2005 Dec; 35(6):1 168-78.

[4] S. Tabbone and L.Wendling., Technical symbols recognition using the two-dimensional Radon transform, In proceedings of the 16 th international Conference on Pattern Recognition Québec (Canada), volume 2, pages 200--203, August 2002

[5] F. Deguillaume, S. Voloshynovskiy and T. Pun, Method for the Estimation and Recovering from General Affine Transform, Proc. SPIE 2002, San Jose, CA, 2002.

[6] Hyung Shin Kim and Heung-Kyu Lee, Invariant Image Watermark using Zernike Moments, IEEE Transactions on Circuits and Systems for Video Technology, Vol.13, No.8, pp.766-775, August 2003

[7] Venkataramana,A.,P.Ananth Raj, 2007, Image Watermarking Using Krawtchouk Moments, International Conference on Computing: Theory and Applications (ICCTA'07), pp. 676-680, March 2007.

[8] A Kadyrov and M Petrou, Affine parameter estimation from the trace transform," IEEE Trans. Pattern Analysis and Machine Intelligence, vol. 28, pp. 1631 - 1645, October 2006.

[9] C. Hundt and M. Liskiewicz., On the complexity of Affine image matching, Symposium on Theoritical Aspects of Computer Science (STACS), 2007

[10] S.R. Deans, The Radon Transform and Some of Its Applications, New York; , 1983. John Wiley \& Sons, Inc. 